\title{
EFFECTS OF AN AGENT INDUCING DOMINANT LETHALS ON RAT SPERM - EXAMINATION WITH ETHYL METHANESULFONATE -
}

\author{
Hironori TAKAGI, Akinori SATOH, Rika SHIRANE, Tomonori HASHIMOTO, \\ Tadahiro INOUE and Masaaki KIMURA
}

\author{
Toxicology Laboratory, Pharmaceutical Research Laboratories, \\ Taisho Pharmaceutical Co., Ltd., \\ 1-403 Yoshino-cho, Omiya-shi, Saitama, 330-8530, Japan
}

(Received July 31, 1999; Accepted December 10, 1999)

\begin{abstract}
Ethyl methanesulfonate (EMS), an alkylating agent which induces dominant lethals, was administered in oral doses of $100 \mathrm{mg} / \mathrm{kg}$ to $\mathrm{Crj}: \mathrm{CD}(\mathrm{SD}) \mathrm{IGS}$ male rats for 5 consecutive days. At the termination of treatment and after a 28-day withdrawal, mating with untreated females and sperm analysis (motion, number, and morphology) were performed. The copulated females were sacrificed at 20 days of gestation.

At the termination of treatment, no clinical signs related to EMS were observed except for a decrease in body weight. Gross pathology and sperm analysis revealed no abnormalities in treated males. However, females mated at the termination of treatment had a clearly higher fetal mortality.

Females mated after the 28-day withdrawal exhibited lower fetal mortality than females mated at the termination of treatment. On the other hand, females mated after the 28-day withdrawal exhibited a lower implantation rate that was not observed in females mated at the termination of treatment. For males after a 28-day withdrawal, sperm analysis revealed both a decrease in sperm motion and number and an increase in morphological change.

These findings indicate that two types of male reproductive toxicity induced by EMS can be distinguished. One induces a low implantation rate that can be detected by sperm analysis, while the other induces fetal lethals that could not be detected by sperm analysis in this study.
\end{abstract}

KEY WORDS: Ethyl methanesulfonate, Dominant lethal, Sperm analysis, IGS rats, CASA, CellSoft4000

\section{INTRODUCTION}

When drugs or other kinds of chemicals were assessed for their effects on male fertility, the importance of sperm analysis attracted a growing interest. Based on the ICH agreement, Japanese guidelines for Reproductive and Developmental Toxicity Studies were amended on September 11 in 1997. The new guidelines included sperm analysis for a study of Fertility and Early Embryonic Development to Implantation. However, appropriate indices of sperm analysis for a study of male fertility are not fully understood. So far, by means of sperm analysis, we have examined the antifertility effects of $\alpha$-chlorohydrin, which induces a decrease in sperm motion without abnormalities in both histopathological examination of the testes and observation of sperm morphology (Yamada et al., 1995). In the present study, we selected ethyl methanesulfonate (EMS), an alkylating agent which induces dominant lethals in rodents. EMS is a mutagen which induces positive reaction in Ames assay (Klopman et al., 1990), UDS assay (van Erp et al., 1992), and Micronucleus assay (Kondo et al., 1989). The dominant lethal effect was reported to continue for 4 weeks in rats at both oral doses of 100 $\mathrm{mg} / \mathrm{kg}$ for 5 consecutive days (McGregor et al., 1983) 
and at an intraperitoneal single dose of $300 \mathrm{mg} / \mathrm{kg}$ (Jackson et al., 1961). It was also reported that the agent induced dominant lethals in rats at an intraperitoneal single dose of $120 \mathrm{~m} / \mathrm{kg}$, but did not induce a decrease in testis weight or histopathological changes in testes for 10 weeks (Murao, 1980). The aim of the present study was to confirm the characteristics of EMS-inducing dominant lethals in Crj:CD(SD)IGS rats and to examine the sperm conditions inducing dominant lethals.

\section{MATERIALS AND METHODS}

\section{Chemicals}

EMS and bovine serum albumin (BSA) were purchased from Sigma-Aldrich (Tokyo, Japan). Dulbecco's phosphate-buffered saline (D-PBS) containing glucose was obtained from Gibco BRL (Tokyo, Japan). All other reagents were purchased from commercial sources and were of at least analytical grade.

\section{Animals}

Crj:CD(SD)IGS rats were purchased from Charles River Japan, Inc. (Tsukuba, Japan). After quarantine and acclimatization, healthy males (12 weeks old) and females ( 11 weeks old) were chosen for experiments. The animal room was maintained at $23 \pm 3^{\circ} \mathrm{C}$, with $50 \pm 20 \%$ relative humidity and a $12 \mathrm{hr}$ light - dark cycle (lights on 7:15 to 19:15). Solid food (MF, Oriental Yeast Inc., Tokyo) and tap water were available ad libitum.

\section{Experimental design}

EMS was prepared freshly each day as a solution in distilled water. Male rats were treated with EMS ( $100 \mathrm{mg} / \mathrm{kg} /$ day, p.o., $0.5 \mathrm{ml} / 100 \mathrm{~g}$ body) once daily for 5 consecutive days. Males in the control group received the solvent alone at the same volume-dosage during the same treatment period. On the day after final administration and after a 28-day withdrawal, the effects of EMS were assessed. The 28-day period is about two cycles of spermatogenesis in testes and enough for spermatozoa to pass through an epididymis. Nine males were mated with untreated virgin females, and 8 other males were autopsied to examine sperm condition. The experimental design is shown in Fig. 1.

\section{Copulation}

Treated males were paired on a one-to-one basis with untreated virgin pro-oestrus females overnight. The following morning, females with sperm detected in the vaginal smear were considered to be at day 0 of gestation. At day 20 of gestation, dams were exsanguinated under ether anesthesia and sacrificed. The numbers of implantations and corpora lutea were counted, and the implantation rate was calculated. Dead fetuses were classified as Resorbed, Early Death, Middle Death, or Late Death according to their size and shape, and the fetal death rate was calculated. Live fetuses were weighed and then examined for sex ratio and external anomalies.

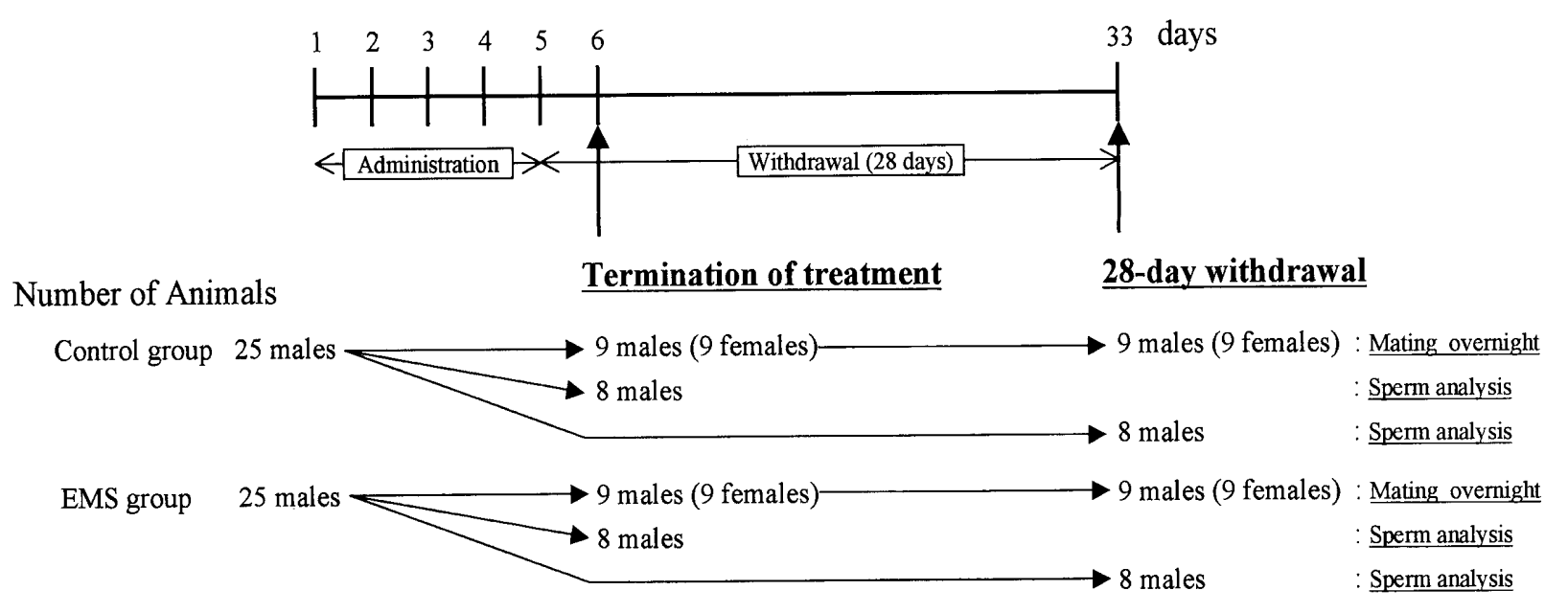

Fig. 1. Experimental design.

Male rats were treated with distilled water or EMS once daily for 5 consecutive days. On the day after final administration (Termination of treatment) and after a 28-day withdrawal (28-day withdrawal), mating and sperm analysis were performed. 
Effects of Ethyl Methanesulfonate on Rat Sperm.

\section{Sperm analysis}

The epididymides were dissected out and weighed. A small part of a caudal epididymis was cut and placed into Petri dishes containing $10 \mathrm{ml}$ of medium $\left(37^{\circ} \mathrm{C}\right.$, DPBS containing $0.5 \% \mathrm{BSA}$ ). After $15 \mathrm{~min}$, the motion of 100 or more sperm was analyzed with the CellSoft4000 computer-assisted sperm analysis system (CASA, CRYO Resources Ltd., NY). The indices calculated by the CASA were percent motile, velocity, amplitude of lateral head displacement (A.L.H.), beat/cross frequency, radius, and percentage of circularly swimming sperm. The other caudal epididymis was weighed and minced in saline $\left(37^{\circ} \mathrm{C}\right)$. After removal of the pieces of organ by filtration, the sperm solution was used for microscopical examination of sperm number and morphology. Sperm number was calculated per weight of caudal epididymis. Sperm morphology was classified as tailless or anomalous.

\section{Statistical analysis}

Data were statistically analyzed using the tests listed below. All quantitative data were tested for equal variance $(\mathrm{p}<0.05)$. If equal variance was found, the data were analyzed by the Student's $t$-test (Yoshimura, 1988). If not, the data were analyzed by Welch's $t$-test (Yoshimura, 1988). Copulation rate, impregnation rate, and sex ratio were analyzed by Fisher's exact probability test (Yoshimura, 1988). Implantation rate and fetal death rate were analyzed by the Wilcoxon test (Yoshimura, 1988). For statistical analysis, fetal parameters served as the experimental units of a dam. All data in the EMS group were compared with data in the corresponding control group. In addition, the fertility data and the findings in dams and fetuses at caesarean section were compared between the day after final administration and after a 28-day withdrawal (Table 1,2). All statistical tests were performed at the 0.05 or 0.01 levels of significance.

\section{RESULTS}

\section{Clinical signs}

No clinical signs related to EMS treatment were observed for either males or females, except for a decrease in body weight. Male body weight decreased soon after EMS treatment (Fig. 2). After the treatment period, body weight in the EMS group recovered in parallel with the trend of body weight in the control group, though the final weight in the EMS group was lower than the weight in the control group.

\section{Copulation}

Both numbers of copulate males and males impregnating were not influenced by EMS treatment (Table 1).

One of the dams copulated with EMS-treated males after a 28-day withdrawal was aborted (Table 2). Decrease in implantation rate was observed only in dams copulating with EMS-treated males after a 28day withdrawal. Dams that copulated with EMS-treated males at the termination of treatment exhibited both a higher rate of fetal death and lower number of live fetuses. Dams copulating with EMS-treated males after a 28-day withdrawal also exhibited both a higher rate of fetal death and lower number of live fetuses, but the fetal death rate was decreased and the number of live fetuses was increased by comparison with dams that copulated with EMS-treated males at the termination of treatment.

There was a lower number of live fetuses accompanied with a higher body weight of live fetuses in dams copulating with EMS-treated males at the termination of treatment.

There were no fetuses with external anomalies in any groups.

\section{Sperm analysis}

At the termination of treatment, weights of testes and of epididymides in the EMS group were not signif-

Table 1. Fertility data.

\begin{tabular}{|c|c|c|c|c|}
\hline & \multicolumn{2}{|c|}{ Termination of treatment } & \multicolumn{2}{|c|}{ 28-day withdrawal } \\
\hline & Control & EMS & Control & EMS \\
\hline No. of male animals & 9 & 9 & 9 & 9 \\
\hline No. of copulate males $\left[\%{ }^{a)}\right]$ & $8[88.9]$ & $8[88.9]$ & $9[100]$ & 9 [100] \\
\hline No. of males impregnating $[\%$ b) & $8[100]$ & $8[100]$ & $9[100]$ & $9[100]$ \\
\hline
\end{tabular}




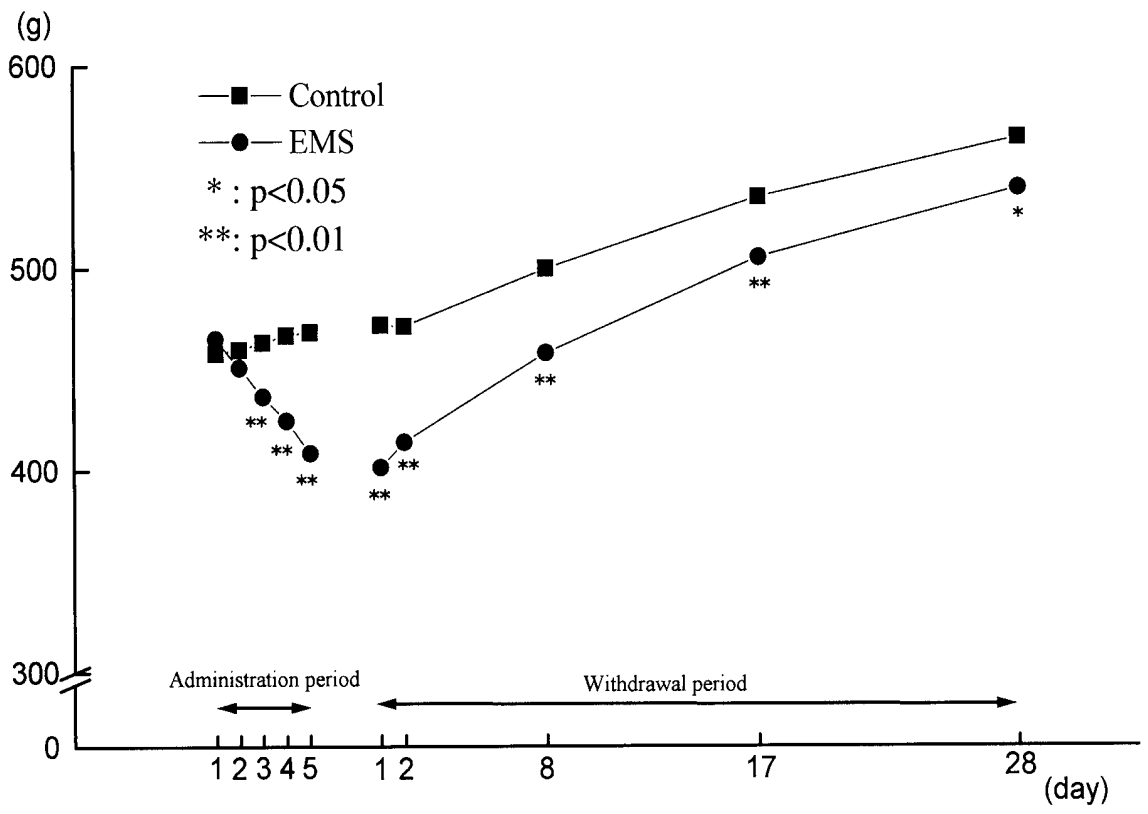

Fig. 2. Body weight changes in males during administration period and withdrawal period.

Table 2. Findings in dams and fetuses at caesarean section.

\begin{tabular}{|c|c|c|c|c|}
\hline & \multicolumn{2}{|c|}{ Termination of treatment } & \multicolumn{2}{|c|}{ 28-day withdrawal } \\
\hline & Control & EMS & Control & EMS \\
\hline No. of dams & 8 & 8 & 9 & 9 \\
\hline No. of females aborted & 0 & 0 & 0 & 1 \\
\hline No. of corpora lutea & $17.0 \pm 2.0$ & $15.4 \pm 3.1$ & $17.9 \pm 3.4$ & $16.0 \pm 2.7$ \\
\hline No. of implantations & $15.1 \pm 2.5$ & $13.6 \pm 2.8$ & $15.3 \pm 2.1$ & $9.2 \pm 4.8^{* * \#}$ \\
\hline Implantation rate (\%) & 89.3 & 88.9 & 87.7 & $56.7^{* * \#}$ \\
\hline No. of fetal deaths & & & & \\
\hline Total $[\%$ a) $]$ & $11[9.2]$ & $85[90.1 * *]$ & $7[5.1]$ & $16\left[22.2^{* * \# \#]}\right.$ \\
\hline Resorbed [\%] & $11[9.2]$ & $84\left[88.9^{* *}\right]$ & $6[4.4]$ & $12[16.2 \#]$ \\
\hline Early death [\%] & $0[0.0]$ & $1[1.2]$ & $1[0.7]$ & $3[5.2]$ \\
\hline Middle death [\%] & $0[0.0]$ & $0[0.0]$ & $0[0.0]$ & $\begin{array}{ll}0 & {[0.0]}\end{array}$ \\
\hline Late death $[\%]$ & $0[0.0]$ & $0[0.0]$ & $0[0.0]$ & $1[0.9]$ \\
\hline Live fetuses & & & & \\
\hline No. of live fetuses & $13.8 \pm 2.9$ & $1.4 \pm 1.3^{* *}$ & $14.6 \pm 2.1$ & $7.4 \pm 4.3^{* *}$ \\
\hline Sex ratio & 1.3 & 1.0 & 0.9 & 0.7 \\
\hline Male/Female & $63 / 47$ & $5 / 5$ & $62 / 69$ & $27 / 40$ \\
\hline Body weight (g) & & & & \\
\hline Male & $3.56 \pm 0.19$ & $3.84 \pm 0.08 *$ & $3.48 \pm 0.46$ & $3.85 \pm 0.38$ \\
\hline Female & $3.39 \pm 0.26$ & $3.85 \pm 0.18 *$ & $3.33 \pm 0.42$ & $3.60 \pm 0.29$ \\
\hline $\begin{array}{l}\text { No. of fetuses with } \\
\text { external anomalies [\%] }\end{array}$ & $0[0.0]$ & $0[0.0]$ & $0[0.0]$ & $0[0.0]$ \\
\hline
\end{tabular}

Mean \pm S.D., ${ }^{\text {a) }}$ : (No. of dead fetus/ No. of implantations) $\times 100$,

Significantly different from the corresponding control value $(* \mathrm{p}<0.05, * * \mathrm{p}<0.01)$,

Significantly different from the EMS group at the termination of treatment $\left(\# p<0.05,{ }^{\#} p<0.01\right)$. 
Effects of Ethyl Methanesulfonate on Rat Sperm.

icantly different from weights of the organs in the control group (Table 3). After a 28-day withdrawal, absolute weights of testes and of epididymides in the EMS group were lower than the control value, but relative weights of the organs were not significantly different from the control values.

At the termination of treatment, the indices of sperm motion in the EMS group were not significantly different from the control values except for the percentage of circularly swimming sperm (Table 4). Sperm number and morphology were not influenced by EMS treatment. After a 28-day withdrawal, some indices of sperm motion were changed by EMS treatment. The percent motile, velocity, A.L.H. max, and A.L.H. mean were lower than those of the control group. Sperm number and morphology were also influenced by EMS treatment. The sperm number was lower and the rate of morphological change was higher than in the control values.

\section{DISCUSSION}

In the examination at the termination of treatment, a high fetal death rate was observed in the EMS-treated

Table 3. Reproductive organ weights in males.

\begin{tabular}{|c|c|c|c|c|}
\hline & \multicolumn{2}{|c|}{ Termination of treatment } & \multicolumn{2}{|c|}{ 28-day withdrawal } \\
\hline & Control & EMS & Control & EMS \\
\hline No. of male animals & 8 & 8 & 8 & 8 \\
\hline Final body weight $(\mathrm{g})$ & $469.6 \pm 28.6$ & $401.9 \pm 21.0^{* *}$ & $585.3 \pm 33.5$ & $545.0 \pm 25.3^{*}$ \\
\hline \multicolumn{5}{|l|}{ Testis } \\
\hline Absolute (g) & $3.25 \pm 0.24$ & $3.19 \pm 0.38$ & $3.66 \pm 0.26$ & $3.10 \pm 0.24 * *$ \\
\hline Relative (\%) & $0.69 \pm 0.05$ & $0.80 \pm 0.12$ & $0.63 \pm 0.05$ & $0.57 \pm 0.05$ \\
\hline \multicolumn{5}{|l|}{ Epididymis } \\
\hline Absolute (g) & $1.27 \pm 0.10$ & $1.14 \pm 0.10$ & $1.40 \pm 0.10$ & $1.24 \pm 0.12^{*}$ \\
\hline Relative (\%) & $0.27 \pm 0.04$ & $0.29 \pm 0.04$ & $0.24 \pm 0.01$ & $0.23 \pm 0.03$ \\
\hline
\end{tabular}

Mean \pm S.D., Significantly different from the corresponding control value $(* p<0.05, * * p<0.01)$.

Table 4. Data from sperm analysis.

\begin{tabular}{|c|c|c|c|c|c|c|c|}
\hline & \multicolumn{3}{|c|}{ Termination of treatment } & \multicolumn{4}{|c|}{ 28-day withdrawal } \\
\hline & Control & \multicolumn{2}{|r|}{ EMS } & \multicolumn{2}{|c|}{ Control } & \multicolumn{2}{|c|}{ EMS } \\
\hline No. of male animals & 8 & & 8 & & 8 & & 8 \\
\hline \multicolumn{8}{|l|}{ Sperm motion } \\
\hline Motile (\%) & $56.0 \pm 13.1$ & 54.9 & \pm 13.6 & 57.5 & \pm 10.7 & 37.8 & $\pm 17.9^{*}$ \\
\hline Velocity $(\mu \mathrm{m} / \mathrm{s})$ & $491.1 \pm 35.8$ & 478.9 & \pm 62.0 & 513.0 & \pm 44.2 & 451.8 & $\pm 46.5^{*}$ \\
\hline Linearity & $2.7 \pm 0.4$ & 2.6 & \pm 0.3 & 2.9 & \pm 0.3 & 2.7 & \pm 0.4 \\
\hline A.L.H. $\quad \max (\mu \mathrm{m})$ & $29.5 \pm 4.2$ & 25.6 & \pm 4.2 & 29.1 & \pm 4.5 & 21.1 & $\pm 5.0^{* *}$ \\
\hline mean $(\mu \mathrm{m})$ & $25.6 \pm 2.7$ & 22.9 & \pm 3.8 & 26.3 & \pm 4.1 & 19.4 & $\pm 5.2^{*}$ \\
\hline Beat/Cross Freq. $(\mathrm{Hz})$ & $8.1 \pm 0.5$ & 8.2 & \pm 0.6 & 8.4 & \pm 1.5 & 9.0 & \pm 3.5 \\
\hline Average Radius $(\mu \mathrm{m})$ & $26.9 \pm 5.8$ & 22.5 & \pm 4.8 & 31.9 & \pm 14.1 & 22.8 & \pm 9.1 \\
\hline Circ. Swim. Cells [per Motile Cells] $(\%)$ & $10.8 \pm 2.1$ & 6.6 & $\pm 2.9 * *$ & 6.1 & \pm 2.8 & 6.5 & \pm 2.6 \\
\hline$[$ [per All Cells] $(\%)$ & $6.0 \pm 1.9$ & 3.6 & $\pm 1.8^{*}$ & 3.5 & \pm 2.1 & 2.4 & \pm 1.3 \\
\hline $\begin{array}{l}\text { Sperm number } \\
\left.\text { ( } \times 10^{8} / 1 \mathrm{~g} \text { distal cauda of epididymis }\right)\end{array}$ & $6.42 \pm 1.01$ & 6.89 & $9 \pm 1.22$ & 6.39 & \pm 0.95 & 3.50 & $\pm 1.11 * *$ \\
\hline \multicolumn{8}{|l|}{ Sperm morphology } \\
\hline Tailless $(\%)$ & $2.53 \pm 1.72$ & 4.49 & $9 \pm 6.27$ & 3.01 & \pm 2.77 & 22.10 & $\pm 16.83 *$ \\
\hline Anomalous (\%) & $0.36 \pm 0.49$ & 0.31 & $1 \pm 0.45$ & 0.31 & \pm 0.60 & 5.37 & $\pm 1.63 * *$ \\
\hline
\end{tabular}

Mean \pm S.D., Significantly different from the corresponding control value $(* \mathrm{p}<0.05, * * \mathrm{p}<0.01)$. 
group, but sperm analysis did not reveal abnormalities except for the percentage of circularly swimming sperm. The percentage of circularly swimming sperm, a sperm motion parameter, was lower than the value of the control group. Concerning this parameter, a report suggests that it may not be appropriate for use to detect alternations in sperm motion because of its wide range of distribution (Kaneto et al., 1999). Our finding about the parameter in this study, moreover, was not reproduced in a subsequent test (not reported). Judging from these facts, we considered that the difference in the percentage of circularly swimming sperm between the control and the EMS-treated group was unrelated to EMS treatment.

In the examination after a 28-day withdrawal, females that copulated with EMS-treated males exhibited a reduced fetal death rate by comparison with females that copulated at the termination of treatment. On the other hand, a decrease in implantation rate was observed, while it was not observed in females that copulated at the termination of treatment. For males treated with EMS, after a 28-day withdrawal, the sperm motion (percent motile, velocity, A.L.H. max and A.L.H. mean) and sperm number were decreased and the rate of morphological change of sperm was increased. Following withdrawal, the implantation rate and sperm condition were worse, while the fetal death rate was lower. Those findings led to a speculation that the decrease in implantation rate was due to the abnormal sperm condition. Decrease in motion and number of sperm may affect fertilization of gametes. However, it is unclear whether the abnormal sperm condition after a 28-day withdrawal affected only the fertilization of gametes or cleavage until implantation. Concerning fertilized gametes, an experiment in which male mice subcutaneously treated with EMS were mated with virgin females and fertilized ova were examined for chromosomal aberrations has been reported (Hashimoto et al., 1987). In this study, fertilized ova taken from females mated with males at 4 weeks or later did not exhibit chromosomal aberrations, while fertilized ova taken from females mated with males within 3 weeks after the treatment did. The results indicate that sperm fertilizing ova at 4 weeks or later after the treatment did not have chromosomal aberrations. Taking this report into consideration, sperm that fertilized ova after 28day withdrawal in our study did not have chromosomal aberrations. The 28-day withdrawal sperm, however, induced the decrease in implantation rate. Judging from the length of the withdrawal period, the 28-day withdrawal sperm were affected by EMS in the period of spermatids in testes; the length of the spermatid period is about 22 days (Takahashi et al., 1994) and spermatozoa need about 11 days to pass through an epididymis (Suzuki, 1989). In the spermatid period, the cells are not matured. As a result of this immatureness, the exposure to EMS in this period probably prevents the cells from growing normally. Sperm abnormalities observed in our sperm analysis, namely decreased number, decreased motion and morphological change, mean death, dysfunction and malformation of sperm, respectively. We consider that sperm having dysfunction and malformation are difficult to fertilize; as a result, fertilized ova do not have chromosomal aberrations.

No abnormality was detected in sperm by our analyses at the termination of EMS treatment, yet the sperm induced high fetal death. It is said that rat sperm stays in a caudal epididymis for 5 days before ejaculation (Suzuki, 1989). Judging from the length of the administration period, the sperm that induced a high fetal death rate at the termination of treatment was exposed by EMS in caudal epididymides. In caudal epididymides, sperm nuclei are condensed and covered with acrosome; as a result, nuclei in this period of spermatogenesis are more tolerant than those in another periods. The sperm of this period, however, induced a high fetal death in our study. In order to investigate the effects of EMS on sperm nuclei, Sega and Owens administered a single dose of tritium-labeled EMS to male mice and counted tritium level in sperm head for 2 weeks (Sega and Owens, 1978 and 1983). In the studies, the tritium level in sperm head exhibited approximately parallel changes with fetal mortality on dominant lethal tests, and the tritium of sperm head was detected not in DNA but in protamine. The results suggest that an analytical method detecting protamine alkylation is useful to assess the potential of chemicals inducing dominant lethals.

With the use of CASA and other methods, we could detect the EMS-inducing inhibition of spermatogenesis. Those findings indicate that sperm analysis using ejaculated sperm without killing of animals can probably detect abnormalities in testes. However, we could not detect EMS-inducing alkylation of sperm head that caused dominant lethals. In order to predict dominant lethals, we have examined several techniques detecting sperm protamine-alkylation. 
Effects of Ethyl Methanesulfonate on Rat Sperm.

\section{REFERENCES}

Hashimoto, T., Takeuchi, K., Ohno, S., Komatsu, S. and Ohno, Y. (1987): Cytogenic analysis of dominant lethal effects of mitomycin $C$ and ethyl methanesulfonate. J. Toxicol. Sci., 12, 33-45.

Jackson, H., Fox, B.W. and Craig, A.W. (1961): Antifertility substances and their assessment in the male rodent. J. Reprod. Fertil., 2, 447-465.

Kaneto, M., Kanamori, S., Hara, K. and Kishi, K. (1999): Characterization of epididymal sperm motion and its correlation with stages of target cells in rats given $\alpha$-chlorohydrin, cyclophosphamide or nitrazepam. J. Toxicol. Sci., 24, 187197.

Klopman, G., Frierson, M.R. and Rosenkranz, H.S. (1990): The structural basis of the mutagenicity of chemicals in Salmonella typhimurium: The GeneTox Data Base. Mutation Res., 228, 1-50.

Kondo, K., Suzuki, H., Hoshi, K. and Yasui, H. (1989): Micronucleus test with ethyl methanesulfonate administered by intraperitoneal injection and oral gavage. Mutation Res., 223, 373-375.

McGregor, D.B., Willins, M.J., McDonald, P., Holmstrom, M., McDonald, D. and Niemeier, R.W. (1983): Genetic effects of 2-methoxyethanol and bis (2-methoxyethyl) ether. Toxicol. and Appl. Pharmacol., 70, 303-316.

Murao, S. (1980): An attempt to induce experimental chromosomal diseases with malformations. Kobe Daigaku Igakubu Kiyo. Journal of the Medical Faculty of Kobe., 41, 141-148.
Sega, A.G. and Owens, J.G. (1978): Ethylation of DNA and protamine by ethyl methanesulfonate in the germ cells of male mice and the relevancy of these molecular targets to the induction of dominant lethals. Mutation Res., 52, 87-106.

Sega, A.G. and Owens, J.G. (1983): Methylation of DNA and protamine by methyl methanesulfonate in the germ cells of male mice. Mutation Res., 111, 227-244.

Suzuki, F. (1989): Handbook of Physiological Sciences (Suzuki, T., Hiwatashi, K. and Kanatani, H., eds.), vol.21, pp.64-71, Physiology of Reproduction, Igaku-Shoin, Japan.

Takahashi, D., Mitsumori, K., Furukawa, F., Inoue, T. and Matsui, H. (1994): Seisoudokuseihyoukanotameno-Seisaikanatorasu (Takahashi, D., ed.), pp.1, Soft Science, Japan.

van Erp, Y.H.M., Koopmans, M.J.E., Heirbaut, P.R.C.M., van der Hoeven, J.C.M. and Weterings, P.J.J.M. (1992): Unscheduled DNA synthesis in human hair follicles after in vitro exposure to 11 chemicals: Comparison with unscheduled DNA synthesis in rat hepatocytes. Mutation Res., 271, 201-208.

Yamada, T., Inoue, T., Sato, A., Yamagishi, K. and Sato, M. (1995): Effects of short-term administration of $\alpha$-chlorohydrin on reproductive toxicity parameters in male Sprague-Dawley rats. J. Toxicol. Sci., 20, 195-205.

Yoshimura, I. (1988): In "Statistical Analysis of Toxicological Data", pp.30-78, Scientist, Tokyo (in Japanese). 